\title{
Sintomatologia musculoesquelética e intensidade dolorosa em varredoras de rua: associação com a capacidade de trabalho
}

\author{
Musculoskeletal symptoms and painful intensity of street sweepers: association with \\ work ability
}

\author{
Josimáteus Geraldo Ataíde Rocha Silva \\ Priscila Almeida Barbosa ${ }^{2}$ \\ José Dionísio de Paula Júnior ${ }^{3}$ \\ Luciano José Minette ${ }^{4}$
}

\section{Resumo}

A varrição integra os serviços de limpeza urbana e, apesar de sua insalubridade, não se conhece bem seu padrão de morbidade. Este estudo tem como objetivo identificar a prevalência relativa aos sintomas osteomusculares, da intensidade da dor e, posteriormente, verificar a associação dessas variáveis com a capacidade de trabalho (CT) de varredoras de rua que prestam serviço de varrição urbana. Foram utilizados, na pesquisa, quatro instrumentos de coleta de dados, sendo uma ficha de anamnese e três questionários, são eles: Questionário Nórdico de Sintomas Osteomusculares (QNSO); a Wong-Baker Faces Pain Rating and Scale (FACES) e o Índice de Capacidade Para o Trabalho (ICT). As regiões prevalentemente mais afetadas foram Quadril/MMII e ombros. Foram encontradas associações entre a CT, a intensidade de dor e a presença de sintomas osteomusculares em distintas regiões do corpo.

Palavras-chave: Limpeza urbana; Ergonomia; Fisioterapia; Epidemiologia; Saúde Ocupacional.

\begin{abstract}
The sweeping integrates urban cleaning services, despite its insalubrity, its pattern of morbidity is not well known. This study aims to identify the prevalence of musculoskeletal symptoms, pain intensity and later to verify these association of variables the work ability (WA) of street sweepers who provide urban sweeping service. Four data collection instruments were used in the study, one anamnesis form and three questionnaires: the Nordic Osteomuscular Symptoms Questionnaire (NMQ); the Wong-Baker Faces Pain Rating and Scale (FACES) and the Work Ability Index (WAI). The predominantly affected regions were Hip / Shoulder and Shoulders. Associations were found between $C T$, pain intensity and the presence of musculoskeletal symptoms in different regions of the body.
\end{abstract}

Keywords: Urban Cleanliness; Ergonomics; Physiotherapy; Epidemiology; Occupational Health.

\footnotetext{
${ }^{1}$ Bacharel em Fisioterapia pela Faculdade Presidente Antônio Carlos de Ubá (FUPAC), licenciando em Dança pela Universidade Federal de Viçosa (UFV), MG, Brasil. E-mail: josimateussilva@gmail.com

${ }^{2}$ Mestre em Saúde coletiva pela Universidade Federal de Juiz de Fora (UFJF), MG, Brasil. Professora Adjunta na Faculdade Presidente Antônio Carlos de Ubá (FUPAC). E-mail: priscilaalmeida_jf@yahoo.com.br

3 Doutor em Ciências Biomédicas pelo Instituto Universitario Italiano de Rosario, IUNIR, Argentina. Professor na Faculdade Presidente Antônio Carlos de Ubá (FUPAC), MG, Brasil. E-mail: dionisiodepaula@yahoo.com.br

4 Doutor em Ciências Florestais pela Universidade Federal de Viçosa (UFV), MG, Brasil. Professor Titular na Universidade Federal de Viçosa (UFV). E-mail: minette@ufv.br
} 


\section{INTRODUÇÃO}

O trabalho de limpeza urbana pode ser considerado um conjunto de atividades que envolvem limpeza e manutenção de espaços públicos, coleta, transporte, descarte e tratamento de lixo urbano, dentre as quais encontra-se o serviço de varrição. A varrição é ofício considerado extenuante, por causa de sua biomecânica gestual, que envolve o manuseio de cargas e a repetição de movimentos, além do risco pela exposição à poeira e aos resíduos químicos, e possibilidade de contaminação por perfurocortantes. Soma-se, ainda, o risco oferecido pelo espaço físico de trabalho, que envolve tanto a topografia, o tipo de calçamento da rua e a exposição ao tempo, tornando o profissional do setor suscetível às agressões típicas do próprio labor (ROBAZZI et al., 1994; SILVEIRA; ROBAZZI; LUIS, 1998; JANINI, 2003; BARBOSA et al., 2010; PATARO; FERNANDES 2014; SOUZA et al., 2016; FUNDACENTRO, 2017; GEMMA; ROJAS; SOARES, 2017; LAZZARI; REIS, 2017).

Nesse contexto, o estudo das condições de saúde do trabalhador e a elaboração de medidas de proteção à sua saúde é de fundamental importância, principalmente em sujeitos do sexo feminino e de idade avançada, uma vez que estudos apontam que as mulheres são mais propensas às doenças ocupacionais quando comparadas ao sexo oposto, e a capacidade laboral declina com o avançar do tempo (SILVA et al., 2010; PAULA et al., 2015; GODINHO et al., 2017). As mulheres atingem o pico de força entre os 25 e 30 anos, entrando em decréscimo da aptidão física a partir dos 55 anos, em função das alterações hormonais decorrentes da menopausa, fato que pode afetar seu desempenho para o trabalho (GALHUE; OZMUN; GOODWAY, 2013). Fortes, Marson e Martinez (2015) apontam que as características neuromusculares, metabólicas e morfológicas femininas colocam as mulheres em desvantagem para a atividade física em relação aos homens. Em seu estudo, elucidam que elas possuem contração muscular menos eficiente, menor concentração de hematócritos e de hemoglobina e maior dificuldade na utilização dos substratos energéticos do corpo: fatores importantes que podem explicar a maior vulnerabilidade do sexo feminino às doenças ocupacionais.

É possível afirmar que existe na sociedade uma divisão sexual do trabalho, na qual as mulheres geralmente são direcionadas às funções semelhantes aos afazeres domésticos e cuidados com a família, evidenciando a importância feminina nos serviços de limpeza (SILVA et al., 2010). Culturalmente, à mulher também são atribuídos os cuidados da casa e a educação dos filhos, tal como uma obrigação não remunerada, o que tende a colocá-las em situação de dupla jornada (FONSECA; FERNANDES, 2010; FRANCO; DRUCK; SELIGMANN-SILVA, 2010; 
SILVA et al., 2010), podendo comprometer a sua capacidade de trabalho (CT) (PAULA et al., 2015).

A capacidade de trabalho pode ser definida como uma medida do envelhecimento funcional, que pode ocorrer anteriormente ao envelhecimento cronológico, caso não haja intervenção, e resulta da interação entre os recursos humanos, mentais, culturais, organizacionais, sociais e o ambiente trabalhista (GODINHO, 2016). A CT expressa o quão bem a saúde do trabalhador se encontra atualmente; como o próprio trabalhador acredita estar a sua aptidão para o trabalho num futuro próximo; e o quão capaz ele é de executá-lo em relação às exigências físicas e mentais de seu trabalho (PAULA et al., 2015). Acrescenta-se, ainda, que a capacidade de trabalho pode ser afetada pela dor ou outros distúrbios musculoesqueléticos, que determinam sinais e sintomas que podem se iniciar de maneira insidiosa e com rápida evolução, caso não haja mudança nas condições laborais (MAGNAGO et al., 2012; GODINHO et al., 2016).

Nesse contexto, os sintomas osteomusculares (SOM), juntamente com a dor e a capacidade de trabalho, podem ser parâmetros úteis na análise da saúde do trabalhador (WALSH et al., 2004; FONSECA; FERNANDES, 2010; PAULA et al., 2015). Contudo, observase escassez de estudos no contexto da saúde do trabalhador que avaliem o padrão de sintomas osteomusculares, a intensidade da dor e a capacidade de trabalho no setor de varrição, de forma concomitante ou isolada. Portanto, este trabalho torna-se relevante à medida que permite conhecer o perfil de saúde e o padrão de morbidade dessa classe trabalhadora, além de apresentar novas diretrizes na elaboração de estratégias de prevenção aos agravos à saúde do trabalhador e medidas ergonômicas mais eficazes, que contemplem as necessidades da atividade de varrição.

Este estudo tem como objetivo identificar a prevalência relativa aos sintomas osteomusculares e de intensidade da dor e, posteriormente, verificar a associação dessas variáveis com a capacidade de trabalho de varredoras de rua que prestam serviço de varrição urbana.

\section{PROCEDIMENTOS METODOLÓGICOS}


Trata-se de um estudo analítico com temporalidade transversal, o qual contempla variáveis de natureza quanti-qualitativas. As informações foram coletadas por um acadêmico do curso de Graduação em Fisioterapia da Fundação Presidente Antônio Carlos FUPAC, Ubá, Minas Gerais.

A pesquisa ocorreu em uma empresa terceirizada, responsável pela limpeza urbana e coleta de resíduos sólidos do município de Ubá-MG. O trabalho cumpriu todos os critérios éticos em acordo com a Resolução n 466/2012 do Conselho Nacional de Saúde, e foi aprovado pelo comitê de ética da FUPAC (09/17).

Participaram da pesquisa somente varredores do gênero feminino. Esse setor conta com 35 profissionais em situação regular, distribuídas nos turnos matutino/vespertino e vespertino/noturno. A amostra foi composta por 21 profissionais, selecionadas a partir dos seguintes critérios de inclusão: ser mulher, ter idade entre 20 a 55 anos, trabalhar na função há no mínimo 18 meses, possuir ausência de afastamentos por mais de trinta dias nos últimos três meses por motivos de saúde e assinar o Termo de Consentimento Livre e Esclarecido (TCLE). Foram excluídas as voluntárias que apresentaram sintomatologia musculoesquelética ou dor recorrente em período anterior à admissão na função; que relataram dor frequente sem relação direta com o ofício; que não responderam uma ou mais questões obrigatórias nos questionários; que atrasaram ou recusaram-se a entregar os instrumentos de avaliação ou Termo de Consentimento Livre e Esclarecido, nas datas previstas.

Esta pesquisa contou com a utilização de uma ficha de anamnese e três instrumentos validados: o Questionário Nórdico de Sintomas Osteomusculares - QNSO (PINHEIRO; TROCCOLI; CARVALHO, 2002), a Escala Wong-Baker Faces Pain Rating Scale - FACES (OLIVEIRA et al., 2014) e o Índice de Capacidade Para o Trabalho - ICT (TUOMI et al., 2005).

A ficha de anamnese foi composta por treze perguntas envolvendo nome, idade, afastamentos, tempo de serviço, doenças prévias à admissão no setor, entre outras questões que abordaram os critérios de participação no estudo. O segundo instrumento aplicado refere-se ao Questionário Nórdico de Sintomas Osteomusculares (QNSO). Segundo os autores, a ferramenta é recomendada para a realização de diagnóstico do posto de trabalho e apresenta confiabilidade para identificação de sintomas osteomusculares, permitindo a padronização de estudos na área, embora careça de recursos para mensurá-los. O QNSO baseia-se em respostas dicotomizadas acerca de nove regiões anatômicas, e investiga a presença de sintomas nos últimos doze meses (SOM12) e nos últimos sete dias (SOM7) (PINHEIRO; TROCCOLI; 
CARVALHO, 2002). A dor, especificamente, foi analisada e considerada por outro instrumento. Após o preenchimento do questionário, foram contabilizadas as regiões afetadas.

A dor foi mesurada nos últimos sete dias pela escala FACES, composta por seis faces cotadas no formato de 0 (sem dor) a 10 (dor máxima), sendo a nota assinalada proporcional à dor (OLIVEIRA et al., 2014; WONG; BAKER, 1988). Em caso de dúvida no preenchimento, a varredora foi orientada a marcar uma das faces apresentadas correspondente à sua dor, passando a ser considerado o valor de número par correspondente à face. Com o intuito de padronizar a intensidade da dor por região, foi utilizada a mesma imagem disponibilizada para o QNSO como feedback visual para as regiões acometidas. O score de dor foi qualificado em ausente (igual a 0), fraca a moderada (1 a 6), forte a insuportável (7 a 10), para, em seguida, ser associado à capacidade de trabalho.

Para mensurar e classificar a capacidade de trabalho, o instrumento aplicado foi o Índice de Capacidade para o Trabalho (ICT). Esse questionário é composto de dez questões, que permitem realizar a avaliação a partir da concepção do próprio trabalhador. As perguntas são divididas em sete domínios, cuja pontuação total varia de 7 (baixa) à 49 (ótima), sendo instrumento confiável para averiguar a saúde laboral (TUOMI et al., 2005; MARTINEZ; LATORRE; FISCHER, 2009; SILVA JÚNIOR, 2010; 2013). Para o presente estudo, a análise da pontuação do ICT foi realizada, conforme sugere Silva Júnior (2010), com dois scores distintos: um para indivíduos abaixo de 35 anos e outro para acima desta idade. As médias dos resultados foram calculadas a fim de verificar o estado geral de capacidade de trabalho do setor. As qualificações da CT foram categorizadas em adequada (boa e ótima - 41 a 49 pontos) e inadequada (baixa e moderada - 7 a 40 pontos).

Os dados colhidos pelos questionários foram organizados por meio do software Statistical Package for the Social Sciences, 21.0 (SPSS). Foram conduzidas análises univariadas, as variáveis categóricas foram submetidas à análise de distribuição de frequência (absoluta e percentual) e as variáveis contínuas submetidas à análise de medidas de tendência central (média) e variabilidade (desvio-padrão), visando descrever as variáveis e os sujeitos da pesquisa. Assim, foram conduzidas as análises de associação bivariada, com o objetivo de verificar a associação entre as variáveis e obter compreensão inicial dos dados coletados com testes Qui-quadrado e $t$-student.

\section{RESULTADOS}


A pesquisa contou com a participação de 21 mulheres que apresentaram idade média de 45 anos, variando entre 21 a 55 anos. O tempo de atuação na empresa foi 4,50 $\pm 2,0$ anos, $71,43 \%$ das participantes não tinham experiência prévia na varrição urbana. Ainda, 80,95\% das trabalhadoras possuíam baixo grau de escolaridade, até a quarta série. Quanto ao número de empregos, 90,48\% trabalhavam apenas na empresa, e, dentre todas as voluntárias desta pesquisa, 85,72\% exerciam atividades domésticas paralelamente ao trabalho. $O$ uso de equipamentos de proteção individual (EPI's) foi unânime durante o expediente de trabalho, sendo os mais citados: a luva, o boné, o uniforme, a capa de chuva e o protetor solar.

Tabela 1- Prevalência topográfica de sintomatologia musculoesquelética em sete dias e em doze meses e associações com a capacidade de trabalho das varredoras de rua

\begin{tabular}{|c|c|c|c|c|c|c|c|}
\hline \multirow[b]{2}{*}{ Região } & \multirow[b]{2}{*}{ Situação } & \multicolumn{2}{|c|}{$\begin{array}{l}\text { Associação entre a } \\
\text { qualificação da CT e } \\
\text { SOM7 }\end{array}$} & \multirow[b]{2}{*}{ P-valor } & \multicolumn{2}{|c|}{$\begin{array}{l}\text { Associação entre a } \\
\text { qualificação da CT e } \\
\text { SOM12 }\end{array}$} & \multirow[b]{2}{*}{ P-valor } \\
\hline & & $\begin{array}{c}\text { Inadequada } \\
\text { n. (\%) }\end{array}$ & $\begin{array}{c}\text { Adequada } \\
\text { n. (\%) }\end{array}$ & & $\begin{array}{l}\text { Inadequada } \\
\text { n. (\%) }\end{array}$ & $\begin{array}{l}\text { Adequada } \\
\text { n. (\%) }\end{array}$ & \\
\hline \multirow[t]{2}{*}{ Pescoço } & Ausente & $5(38,5)$ & $8(61,5)$ & 0,104 & $5(45,5)$ & $6(54,5)$ & 0,505 \\
\hline & Presente & $6(75,0)$ & $2(25,0)$ & & $6(60,0)$ & $4(40,0)$ & \\
\hline \multirow[t]{2}{*}{ Ombro †• } & Ausente & $3(27,3)$ & $8(72,7)$ & $0,016^{*}$ & $2(33,3)$ & $4(66,7)$ & 0,269 \\
\hline & Presente & $8(80,0)$ & $2(20,0)$ & & $9(60,0)$ & $6(40,0)$ & \\
\hline \multirow[t]{2}{*}{ Braço †• } & Ausente & $4(36,4)$ & $7(63,6)$ & 0,123 & $3(37,5)$ & $5(62,5)$ & 0,284 \\
\hline & Presente & $7(70,0)$ & $3(30,0)$ & & $8(61,5)$ & $5(38,5)$ & \\
\hline \multirow[t]{2}{*}{ Cotovelo } & Ausente & $9(52,9)$ & $8(47,1)$ & 0,916 & $9(52,9)$ & $8(47,1)$ & 0,916 \\
\hline & Presente & $2(50,0)$ & $2(50,0)$ & & $2(50,0)$ & $2(50,0)$ & \\
\hline \multirow[t]{2}{*}{ Antebraço } & Ausente & $6(40,0)$ & $9(60,0)$ & 0,072 & $5(38,5)$ & $8(61,5)$ & 0,104 \\
\hline & Presente & $5(83,3)$ & $1(16,7)$ & & $6(75,0)$ & $2(25,0)$ & \\
\hline \multirow[t]{2}{*}{ Punhos mãos e dedos } & Ausente & $6(50,0)$ & $6(50,0)$ & 0,801 & $3(30,0)$ & $7(70,0)$ & $0,050^{*}$ \\
\hline & Presente & $5(55,6)$ & $4(44,4)$ & & $8(72,7)$ & $3(27,3)$ & \\
\hline \multirow[t]{2}{*}{ Região Dorsal } & Ausente & $5(35,7)$ & $9(64,3)$ & $0,031^{*}$ & $4(40,0)$ & $6(60,0)$ & 0,279 \\
\hline & Presente & $6(85,7)$ & $1(14,3)$ & & $7(63,6)$ & $4(36,4)$ & \\
\hline \multirow[t]{2}{*}{ Região Lombar • } & Ausente & $5(35,7)$ & $9(64,3)$ & $0,031^{*}$ & $4(50,0)$ & $4(50,0)$ & 0,864 \\
\hline & Presente & $6(85,7)$ & $1(14,3)$ & & $7(53,8)$ & $6(46,2)$ & \\
\hline \multirow[t]{2}{*}{ Quadril e MMII †• } & Ausente & $5(45,5)$ & $6(54,5)$ & 0,505 & $2(28,6)$ & $5(71,4)$ & 0,122 \\
\hline & Presente & $6(60,0)$ & $4(40,0)$ & & $9(64,3)$ & $5(35,7)$ & \\
\hline
\end{tabular}

Fonte: $O$ autor.

Legenda: *Significante ao nível de $<5 \%$. †Regiões com maior número de acometimentos em FSOM7. - Regiões com maior número de acometimentos em FSOM12. MMII: Membros inferiores. 
Nos últimos sete dias, $76,19 \%$ das trabalhadoras apresentaram sintomatologia musculoesquelética. As principais prevalências ocorreram igualmente nas regiões de ombro, braço e quadril/MMII (47,61\%), e 95,2\% das varredoras apresentaram algum evento sintomático nos últimos doze meses, sendo as regiões mais prevalentes: o quadril/MMII (71,46\%), seguida de ombro $(66,66 \%)$, braço $(38,09 \%)$ e região lombar $(38,09 \%)$, conforme percebido ao se analisar as prevalências topografadas na tabela 1.

Conforme se pode verificar na tabela 2, foram identificadas as prevalências de dor por região nos últimos sete dias, sendo as principais nas regiões de quadril/MMII $(61,9 \%)$, braço $(52,4 \%)$ e ombro $(42,9 \%)$.

Tabela 2 - Prevalência de dor por região topografada encontrada no setor de varrição urbana nos últimos sete dias

\begin{tabular}{|c|c|}
\hline Presença de dor por região & $\mathbf{n}(\%)$ \\
\hline Pescoço & $8(38,1)$ \\
\hline Ombro & $9(42,9)$ \\
\hline Braço & $11(52,4)$ \\
\hline Cotovelo & $4(19,0)$ \\
\hline Antebraço & $7(33,3)$ \\
\hline Punhos mãos e dedos & $9(42,9)$ \\
\hline Região Dorsal & $8(38,1)$ \\
\hline Região Lombar & $9(42,9)$ \\
\hline Quadril e MMII & $13(61,9)$ \\
\hline
\end{tabular}

Fonte: $O$ autor.

$\mathrm{Na}$ tab. 3, evidencia-se a estratificação dos níveis de dor. Considerando-se as regiões afetadas por acometimentos severos, percebeu-se que as prevalências foram ombro (14,28\%), quadril/MMII (9,52\%), antebraço (9,52\%), punhos, mãos e dedos (9,52\%) e braço (4,76\%). A intensidade da dor analisada pela escala de FACES apresentou uma média de 2,09 \pm 0,62, sendo classificada como fraca a moderada após categorização (score de 1 a 6). 
Tabela 3 - Prevalências e associação entre os níveis de dor nos últimos sete dias por região topografada e a capacidade de trabalho das voluntárias do serviço de varrição urbana em frequência absoluta e relativa

\begin{tabular}{|c|c|c|c|c|}
\hline Região & Situação & $\begin{array}{c}\text { CT inadequada } \\
\text { n. }(\%)\end{array}$ & $\begin{array}{c}\text { CT adequada } \\
\text { n. }(\%)\end{array}$ & P-valor \\
\hline \multirow{3}{*}{ Pescoço } & Ausente & $5(38,5)$ & $8(61,5)$ & \multirow{3}{*}{0,230} \\
\hline & Fraca a moderada & $5(71,4)$ & $2(28,6)$ & \\
\hline & Forte a insuportável & $1(100,0)$ & $0(0,0)$ & \\
\hline \multirow{2}{*}{ Ombro r } & Ausente & $3(25,0)$ & $9(75,0)$ & \multirow{2}{*}{$0,013^{*}$} \\
\hline & Fraca a moderada & $5(83,3)$ & $1(16,7)$ & \\
\hline & Forte a insuportável & $3(100,0)$ & $0(0,0)$ & \\
\hline \multirow{3}{*}{ Braço } & Ausente & $3(30,0)$ & $7(70,0)$ & \multirow{3}{*}{0,125} \\
\hline & Fraca a moderada & $7(70,0)$ & $3(30,0)$ & \\
\hline & Forte a insuportável & $1(100,0)$ & $0(0,0)$ & \\
\hline \multirow{3}{*}{ Cotovelo } & Ausente & $9(52,9)$ & $8(47,1)$ & \multirow{3}{*}{0,510} \\
\hline & Fraca a moderada & $2(66,7)$ & $1(33,3)$ & \\
\hline & Forte a insuportável & $0(0,0)$ & $1(100,0)$ & \\
\hline \multirow{3}{*}{ Antebraço + } & Ausente & $5(35,7)$ & $9(64,3)$ & \multirow{3}{*}{$0,047^{*}$} \\
\hline & Fraca a moderada & $5(100,0)$ & $0(0,0)$ & \\
\hline & Forte a insuportável & $1(50,0)$ & $1(50,0)$ & \\
\hline \multirow{3}{*}{ Punhos, mãos e dedos } & Ausente & $5(41,7)$ & $7(58,3)$ & \multirow{3}{*}{0,455} \\
\hline & Fraca a moderada & $5(71,4)$ & $2(28,6)$ & \\
\hline & Forte a insuportável & $1(50,0)$ & $1(50,0)$ & \\
\hline \multirow{3}{*}{ R. Dorsal } & Ausente & $5(38,5)$ & $8(61,5)$ & \multirow{3}{*}{0,230} \\
\hline & Fraca a moderada & $5(71,4)$ & $2(28,6)$ & \\
\hline & Forte a insuportável & $1(100,0)$ & $0(0,0)$ & \\
\hline \multirow{3}{*}{ Região Lombar } & Ausente & $5(41,7)$ & $7(58,3)$ & \multirow{3}{*}{0,409} \\
\hline & Fraca a moderada & $5(62,5)$ & $3(37,5)$ & \\
\hline & Forte a insuportável & $1(100,0)$ & $0(0,0)$ & \\
\hline \multirow{3}{*}{ Quadril e MMII + } & Ausente & $4(50,0)$ & $4(50,0)$ & \multirow{3}{*}{0,249} \\
\hline & Fraca a moderada & $7(63,6)$ & $4(36,4)$ & \\
\hline & Forte a insuportável & $0(0,0)$ & $2(100,0)$ & \\
\hline
\end{tabular}

Fonte: $O$ autor.

Legenda: *Significante ao nível de $<5 \%$. + Regiões com mais casos de dor intensa. MMII: Membros inferiores. 
$\mathrm{Na}$ Tab. 4, a CT prevalente entre as voluntárias foi classificada como inadequada $(52,4 \%)$ pelo ICT. Por sua vez, o setor de varrição urbana apresentou score médio de $38,19 \pm$ 6,73 , qualificando a CT do setor em questão também como inadequada $(<40)$.

Tabela 4 - Análise da prevalência e qualificação da CT das varredoras do setor de varrição urbana da empresa

\begin{tabular}{l|c}
\hline \multicolumn{2}{c}{ Classificação da CT dentre as voluntárias (\%) } \\
\hline Adequada & $47,6 \%$ \\
\hline Inadequada & $52,4 \%$ \\
\hline \multicolumn{2}{c}{ Qualificação da CT do setor de varrição urbana } \\
\hline CT do setor & Valor médio e desvio padrão \\
\hline Inadequada & $38,19 \pm 6,73$ \\
\hline \multicolumn{2}{c}{ Autopercepção da CT pelas varredoras } \\
\hline Igual ou superior a 80\% da melhor fase da vida & $80,95 \%$ \\
\hline
\end{tabular}

Fonte: $\mathrm{O}$ autor.

A associação entre a capacidade de trabalho e a prevalência topográfica de sintomas osteomusculares em sete dias, conforme observado na tabela 1, foi analisada pelo teste de Quiquadrado. Foram constatadas associações entre a $\mathrm{CT}$ e as regiões topográficas de ombro $(p=0,016)$, região dorsal $(p=0,031)$ e região lombar $(p=0,031)$, indicando que a presença dos sintomas nessas regiões diminui a capacidade de trabalho. Não houve associações entre SMO12 e a CT no presente estudo.

As associações entre a intensidade de dor e a CT, identificadas na tabela 3, por sua vez, apresentam-se pelas análises bivariadas, nas quais verificou-se que, dentre todas as regiões avaliadas, o ombro $(p=0,013)$ e o antebraço $(p=0,047)$ apresentaram associação com a CT. Observou-se que há grande tendência de as voluntárias acometidas nessas regiões apresentarem CT inadequada. 


\section{DISCUSSÃO}

As prevalências de sintomas osteomusculares observadas neste estudo acometeram $95,2 \%$ das trabalhadoras nos últimos doze meses e 76,19\% nos últimos sete dias, o que sugere a necessidade eminente de cuidados com essa população, uma vez que tais fatores são preditores de risco para DORT (Distúrbios Osteomusculares Relacionados ao Trabalho) (FRANCO; DRUCK; SELIGMANN-SILVA, 2010; MAGNAGO et al., 2012; CARDOSO; ROMBALDI; SILVA, 204;). Os scores atingidos na análise do ICT do setor e de forma individual também acusaram risco de adoecimento (SILVA JÚNIOR, 2010; SILVA JÚNIOR, 2013). No entanto, a autopercepção da saúde laboral não foi condizente com a real situação, sugerindo que as varredoras desconhecem seu estado de saúde.

As regiões do ombro e quadril/MMIl destacaram-se no que diz respeito às prevalências de dor, intensidade de dor, frequência de sintomas em sete dias e em doze meses, estando sempre entre uma das três regiões mais acometidas. Semelhantemente, Martarello e Benatti (2009) também observaram o ombro como a região prevalente para a presença de sintomas osteomusculares em trabalhadores da higiene e limpeza hospitalar. Souza et al. (2010), utilizando também o QNSO, encontraram as prevalências de sintomas osteomusculares em joelhos, ombro e coluna lombar, ao analisarem 31 servidores de um hospital público, considerando os últimos doze meses.

A prevalência de sintomas musculoesqueléticos em membros superiores apontada neste estudo, especialmente nas regiões de ombro e braço, pode ser justificada pela alta taxa de repetição de movimentos, assim como pela biomecânica gestual característica da função de varrer, além do manuseio de carga, elevação de membros superiores e colocação do lixo no carrinho. Fonseca e Fernandes (2010) também encontraram maior prevalência de sintomas na região de ombro em um estudo com 320 profissionais da enfermagem, além de associação entre a presença de sintomas e a demanda física (repetitividade e força). Essa associação pode ser encontrada também em material disponibilizado sobre LER e DORT pelo Ministério da Saúde (BRASIL, 2012).

No que se refere à prevalência de sintomas em quadril/MMII encontrados em nosso estudo, Gemma, Rojas e Soares (2017) apontam em seu trabalho resultados equivalentes quanto à prevalência nessas regiões, em agentes de limpeza terceirizados de uma faculdade pública. Souza et al. (2010) identificaram o joelho e o ombro dentre as três regiões mais prevalentes acometidas por sintomas musculoesqueléticos em trabalhadores de um hospital, de 
maneira semelhante ao nosso. Cardoso, Rombaldi e Silva (2014) utilizaram uma versão alternativa do QNSO para investigar a presença de sintomas osteomusculares em 127 coletores de lixo, e identificaram a prevalência em pernas, joelho e lombar. Acredita-se que a justificativa para a alta prevalência encontrada nesse estudo esteja associada a fatores, tal como o uso de calçados sem amortecimento adequado e longas caminhadas (CARDOSO; ROMBALDI; SILVA, 2014), somados à topografia da cidade (SANTOS et al., 2009) e à manutenção do ortostatismo por longos períodos (SILVA et al., 2010), o trabalho em pé e o transporte de materiais pesados (FRANCO; DRUCK; SELIGMANN-SILVA, 2010).

Contrariamente ao esperado, o score médio de dor encontrado no setor da empresa, avaliado pela escala FACES, apresentou-se qualificado como dor fraca a moderada $(2,09 \pm$ 0,62), possivelmente em virtude da frequente autoprescrição de analgésicos e miorrelaxantes percebidos nas conversas ocorridas durante a aplicação dos instrumentos. As regiões prevalentes acometidas pela dor foram quadril/MMII (61,9\%), braço (52,4\%), ombro $(42,9 \%)$, punho, mãos e dedos (42,9\%) e coluna lombar (42,9\%). Quando analisadas as regiões com maior intensidade dolorosa, foram identificadas as regiões do ombro e quadril/MMII (14,28\%), antebraço, punhos, mãos e dedos (9,52\%).

Dessa forma, concluiu-se que as regiões mais afetadas pela dor se mantêm dentre as de maior severidade, quando comparadas. Contudo, a mesma sequência de prevalências se altera quando analisada segundo a intensidade de dor de nível forte a insuportável. De modo semelhante ao presente estudo, Lima Júnior e Silva (2014) analisaram a presença de sintomas musculoesqueléticos e dor em 49 docentes, através do-QNSO e da Escala Analógica Visual, e encontraram as maiores prevalências sintomáticas na região lombar (54,8\%), cervical (45,2\%) e ombros $(23,8 \%)$ e de dor na região dos membros superiores. Não foram encontrados estudos que padronizassem e investigassem as prevalências de dor comparando-as posteriormente às regiões de prevalência de dor mais intensa.

A CT do setor analisado foi classificada como inadequada. Silva, Haddad e Domansky (2010) descreveram as intervenções necessárias, segundo a classificação da CT, sugerindo que a CT moderada deve ser melhorada e a baixa deve ser restaurada. Este estudo, entretanto, reuniu as categorias "baixa" e "moderada" e as designou como "CT inadequada", conforme observado por Godinho et al. (2017) e Paula et al. (2015). Desse modo, embasado no score atingido, na categorização da CT e em concordância com todos os autores supracitados, observa-se a necessidade de intervenção em prol da melhoraria e do monitoramento da CT das varredoras do setor, uma vez que a autopercepção da CT não se mostrou medida confiável. Por 
isso, com base em outros autores (SILVA et al., 2010; SZNELWAR, UCHIDA; LANCMAN, 2011; ASSUNÇÃO; ABREU, 2017), sugere-se as seguintes intervenções: implementação de serviço de identificação precoce e diagnóstico de doenças ou convênios de saúde, incentivo à prática regular de exercícios, educação em saúde e prevenção de acidentes, e também a ginástica laboral, orientada por um profissional e com fins terapêuticos (SILVA et al., 2010).

A presença de sintomas osteomusculares em sete dias associou-se, significativamente, à CT nas regiões do ombro, coluna dorsal e lombar, sugerindo que trabalhadoras que possuem sintomatologia nessas regiões tendem a ter sua capacidade de trabalho comprometida. Concordantemente, estudos prévios (SILVA et al., 2010; MAGNAGO et al., 2012; SEGHETTO; PICCOLI, 2012) demonstraram que a presença de sintomas osteomusculares se associa à redução da CT. Não foram encontradas associações significantes entre SMO12 e a CT. Este estudo se diferenciou dos demais por investigar as associações tomando como base diferentes regiões do corpo.

As análises bivariadas de dor e CT apresentaram associações significantes $(p<0,05)$ nas regiões topográficas de ombro e antebraço, estando a presença de dor associada à CT inadequada. Utilizando metodologia semelhante a do presente estudo, Magnago et al. (2012) avaliaram a intensidade de dor pela Escala Numérica de Dor e a capacidade de trabalho por intermédio do ICT, em 592 trabalhadores de enfermagem de um hospital público, e também encontraram associação entre dor e CT. Os autores, entretanto, consideraram o maior score de dor percebido, não considerando as zonas topográficas, como avaliado neste estudo. Outros estudos, com distintas metodologias, também encontraram associação entre a dor e a CT (MASSON, MONTEIRO; VEDOVATO, 2015; PAULA et al., 2015).

As associações encontradas neste estudo entre a CT, a dor e os SOM estão em consonância aos achados de Magnago et al. (2012), Serranheira et al. (2012) e Paula et al. (2015), que reforçam a concepção dessas variáveis enquanto indicadores de excesso de solicitação muscular, ou seja, tratam-se de sinais de alerta para o eminente potencial risco de doenças ocupacionais (BRASIL, 2012). Este artigo também contempla os DORT's enquanto eventos multifatoriais, tal como é preconizado na literatura (GOMES; OLIVEIRA, 2013; MARTINS; FELLI, 2013; GODINHO, 2016), sem desconsiderar o fator de overuse do sistema musculoesquelético pelo levantamento das condições de saúde mediado pelo ICT, escala de FACES e o QNSO. A análise obtida reforça a necessidade de uma aprofundada investigação quando se refere ao estado de saúde do trabalhador. 


\section{CONCLUSÃO}

As avaliações realizadas pelo QNSO, ICT e pela escala FACES demonstraram elevado índice de SOM e dor. As regiões, prevalentemente, mais afetadas foram Quadril/MMII e ombros. A CT inadequada se fez dominante na maioria das profissionais. Foram encontradas associações entre a CT, a intensidade de dor e a presença de sintomas osteomusculares em distintas regiões do corpo, sugerindo que a dor e outros sintomas musculoesqueléticos, em regiões delimitadas, impactam negativamente na CT.

Nesse contexto, percebe-se que há muito a ser feito, principalmente no que se refere a intervenções na saúde do trabalhador, por medidas que favoreçam o rastreamento e diagnóstico precoce de Distúrbios Osteomusculares Relacionados ao trabalho (DORT).

\section{REFERÊNCIAS}

ASSUNÇÃO, A. A.; ABREU, M. N. S. Factor associated with self-reported work-related musculoskeletal disorders in Brazilian adults. Rev Saúde Pública. v. 51, n. 1, p. 01-10, 2017. scrosset Goegle

BARBOSA, S. C. et al. Perfil de Bem-Estar Psicológico em Profissionais de Limpeza Urbana. Rev psicolorgan trab. v. 10, n. 2, p. 54-66, 2010. Google

BRASIL, Ministério da Saúde. Dor relacionada ao trabalho Lesões por esforços repetitivos (LER) Distúrbios osteomusculares relacionados ao trabalho (DORT). 1. Ed. Editora: Editora do Ministério da Saúde, 2012.

CARDOSO, R. K.; ROMBALDI, A. J.; SILVA M. C. Distúrbios osteomusculares e fatores associados em coletores de lixo de duas cidades de porte médio do sul do Brasil. Rev dor. $v$. 15, n. 1, p. 13-17, 2014.

FONSECA, N. R.; FERNANDES, R. C. P. Fatores associados a distúrbios musculoesqueléticos em trabalhadoras de enfermagem. Rev Latino-AM de enfermagem. v. 18, n. 6, p. 1076-1083, 2010.

FORTES, M. S.; MARSON, R. A., MARTINEZ, E. C. Comparação de desempenho físico entre homens e mulheres: Revisão de literatura. Rev Min Educ Fís. v. 23, n. 2, p. 54-69, 2015. Google

FRANCO, T.; DRUCK, G.; SELIGMANN-SILVA, E. As novas relações de trabalho, o desgaste mental do trabalhador e os transtornos mentais no trabalho precarizado. Rev bras saúde ocup. v. 35, n. 122, p. 229-248, 2010. Fcrouarut Google

\section{FUNDACENTRO. Audiência Pública sobre Norma Regulamentadora para os}

Trabalhadores da Limpeza Urbana. Disponível em: <http://www.fundacentro.gov.br/cursos-e- 
eventos/detalhe-do-evento/2017/3/audiencia-publica-sobre-norma-regulamentadora-para-ostrabalhadores-da-limpenza-urbana>. Acesso em: 27 maio 2017.

GALLAHUE, D. L.; OZMUN, J. C.; GOODWAY, J. D. Compreendendo o Desenvolvimento Motor de bebês, crianças, adolescentes e adultos. 3.ed. Editora: AMGH Editora Ltda., 2013. Google

GEMMA, S. F. B.; ROJAS, M. F.; SOARES, M. J. B. Agentes de limpeza terceirizados: entre o ressentimento e o reconhecimento. Rev bras saúde ocup. v. 42, n. 4, p. 1-10, 2017. Zcrourut Google

GODINHO, M. R. et al. Work ability and associated factors of Brazilian technical-administrative workers in education. BMC Res Notes. v. 9, n. 1, 2016. Zcrosset Google

GODINHO, M. R.; FERREIRA, A. P.; FAYER, V. A.; BOFATTI, R. J.; GRECO, R. M. Work ability and associated factors among professionals in Brazil. Rev bras med trab. v. 15, n. 1, p. 88-100, 2017. Google

GOMES, C. C.; OLIVEIRA, R. S. Agentes de limpeza pública: um estudo sobre a relação prazer/sofrimento no ambiente laboral. Psicologia: Ciência e Profissão. v. 33 n. spe, p. 138153, 2013. Ycrosser Google

JANINI, J. P. Fatores de risco e agravo à saúde dos varredores de rua na perspectiva da enfermagem. 2003. Dissertação (Mestrado em Enfermagem). Universidade do Estado do Rio de Janeiro. Rio de Janeiro, 2003. Google

LAZZARI, M. A.; REIS, C. B. Os coletores de lixo urbano no município de Dourados (MS) e sua percepção sobre os riscos biológicos em seu processo de trabalho. Ciênc saúde coletiva. v. 16, n. 8, p. 437-344, 2017. Fcrossert Google

LIMA JÚNIOR, J. P.; SILVA, T. F. A. Análise da sintomatologia de distúrbios osteomusculares em docentes da Universidade de Pernambuco - Campus Petrolina. Rev Dor. v. 15, n. 4, p. 276-280, 2014. Google

MAGNAGO, T. S. B. S. et al. Intensidade da dor musculoesquelética e a (in)capacidade para o trabalho na enfermagem. Rev Latino-Am Enfermagem. v. 20, n. 6, p. 9-18, 2012. Google

MARTARELLO, N. A.; BENATTI, M. C. C. Qualidade de vida e sintomas osteomusculares em trabalhadores da higiene hospitalar. Rev Esc Enferm USP. v. 43, n. 2, p. 419-425, 2009. ₹crosset Google

MARTINEZ, M. C.; LATORRE, M. R. D. O.; FISCHER, F. M. Validade e confiabilidade da versão brasileira do Índice de Capacidade para o Trabalho. Rev Saúde Pública. v. 43, n. 3, p. 525-532, 2009. "Bcrosset

MARTINS, A. C.; FELLI, V. E. A. Sintomas músculo-esqueléticos em graduandos de enfermagem. Enfermagem em Foco. v. 4, n. 1, p. 58-62, 2013. "crosser Google 
MASSON, V. A.; MONTEIRO, M. I.; VEDOVATO, T. G. Trabalhadores da CEASA: fatores associados à fadiga e capacidade para o trabalho. Rev Bras Enferm. v. 68, n. 3, p. 460-466, 2015.

OLIVEIRA, A. M. et al. A functional analysis of the Wong-Baker Faces Pain Rating Scale: linearity, discriminability and amplitude. Rev Enf Ref. v. 4, n. 3, p. 121-130, 2014. Scorosest Google

PATARO, S. S. M.; FERNANDES, R. C. P. Heavy physical work and low back pain: the reality in urban cleaning. Rev Bras Epidemiol. v. 17, n. 1, p. 17-30, 2014. Fcomarut Google

PAULA, I. R. et al. Work ability, musculoskeletal symptoms and quality of life among community health workers in Uberaba, Minas Gerais, Brazil. Rev soc. v. 24, n. 1, p. 152-164, 2015.

PINHEIRO, F. A.; TROCCOLI, B. T.; CARVALHO, C. V. Validação do Questionário Nórdico de Sintomas Osteomusculares como medida de morbidade. Rev Saúde Pública. v. 36, n. 3, p. 307- 312, 2002. Ficourat Google

ROBAZZI, M. L. C. C. et al. O Serviço dos Coletores de Lixo: riscos ocupacionais versus agravos a saúde. Rev Esc Enferm USP. v. 28, n. 2, p.177-189, 1994. "scourat Google

SANTOS, M. C. O. et al. Desregulamentação do trabalho e desregulação da atividade: o caso da terceirização da limpeza urbana e o trabalho dos garis. Prod. v. 19, n. 1, p. 202-2013, 2009. scrourar Google

SEGHETTO, A.; PICCOLI, J. C. J. Nível de atividade física, prevalência de desconforto e dor muscular e capacidade de trabalho: uma avaliação no setor de callcenterde um banco do Rio Grande do Sul, Brasil. Rev Bras cienc e Mov. v. 20, n. 3, p. 105-117, 2012. Goggle

SERRANHEIRA, F. et al. Lesões musculoesqueléticas ligadas ao trabalho em enfermeiros portugueses: ossos do ofício ou doenças relacionadas com o trabalho?. Rev Port Saúde Pública. v. 30, n. 2, p. 193-203, 2012. ‘crowart Google

SILVA JÚNIOR, S. H. A. Avaliação de qualidades psicométricas da versão brasileira do Índice de Capacidade para o Trabalho. 2010. Dissertação (Mestrado em Epidemiologia em Saúde Pública). Fundação Osvaldo Cruz, Escola Nacional de Saúde Pública, Rio de Janeiro, 2010. Google

SILVA JÚNIOR, S. H. A. et al. Test-retest reliability of the Work Ability Index (WAI) in nursing workers. Rev bras epidemiol. v. 16, n. 1, p. 202-209, 2013. Google

SILVA, L. G. et al. Capacidade para o trabalho entre trabalhadores de higiene e limpeza de um hospital universitário público. Rev Eletr Enf. v. 12, n. 1, p. 158-163, 2010. Zcorosest Google

SILVEIRA, E. A. A.; ROBAZZI, M. L. C. C.; LUIS M. A. V. Varredores de rua: acidentes de trabalho ocorridos na cidade de Ribeirão Preto. Rev latino-am enfermagem. v. 6, n.1, p. 7179, 1998. Scrossert Google 
SOUZA, D. B. O. et al. Capacidade para o trabalho e sintomas osteomusculares em trabalhadores de um hospital público. Fisioter Pesqui. v. 22, n. 2, p. 182-190, 2015. Googgle

SOUZA, M. N. A. et al. Estresse, qualidade de vida e trabalho: estudo com agentes da limpeza urbana. Rev bras Qual Vida. v. 8, n. 4, p. 281-295, 2016.

SZNELWAR, L. I.; UCHIDA, S.; LANCMAN, S. A subjetividade no trabalho em questão. Tempo Social. v. 23, n. 1, p. 11-30, 2011.

TUOMI, K. et al. Índice de capacidade para o trabalho. São Carlos: EDUFSCAR; 2005.

WALSH, I. A. P. et al. Capacidade para o trabalho em questões com lesões músculoesqueléticas crônicas. Rev Saúde Pública. v. 38, n. 2, p. 149-156, 2004. „roosest Google

WONG, D. L.; BAKER, C. M. Pain in children: Comparison of assessment scales. Pediatr Nurs. v. 14, n. 1, p. 9-17, 1988. Google 